the intensity of background radiation. The decrease in number of kicks ( $b$ in Fig. 1) corresponds to the lower limit of $\gamma$-ray emission, from which wo conclude that the lower limit of the cross-section in question is $0.09 \times 10^{-4}$ times that for the cadmium atom.

Dunning and Pegram ${ }^{3}$ have obtained the value $3,300 \times 10^{-24} \mathrm{~cm} .^{2}$ for the absolute scattering crosssection of cadmium atoms. Assuming that this is equal to the eross-section for the process of capture ${ }^{4}$ accompanied by the $\gamma$-ray emission, we can locate the cross-section for the recombination of neutron with proton as lying between $8.3 \times 10^{-26} \mathrm{~cm}^{2}$ and $3.0 \times 10^{-26} \mathrm{~cm} .^{2}$.

Our method is free from any ambiguous assumptions, and the correctness of the values depends mainly upon the value of the cross-section of cadmium atoms, the determination of which can be carried out fairly accurately. We believe, therefore, that our values are correct within an error of twenty per cent, though they are much lower than those obtained by other investigators with different methods. Details will be published soon in the Proc. Phys.-Math. Soc. Jap.

$\begin{array}{ll} & \text { S. KIKUCHI. } \\ & \text { K. HUSIMI. } \\ \text { Osaka Imperial University, } & \text { H. AoKI. }\end{array}$
Japan.

Nov. 10.

${ }^{1}$ S. Kikuchi, H. Aoki and K. Husimi, Proc. Phys.-Math. Soc. Jap., 17, 369 (1935). Proc. Imp. Acad. Jap., 11, 253 (1935).

${ }_{3}$ J. R. Dunning, G. B. Pegram, G. A. Fink and D. P. Mitchell, Phys. Rev., 47, 970 .

J. R. Dunning and G. B. Pegram, Phys. Rev., 48, 265 (1935).

\section{Angular Momentum of Circularly Polarised Light}

By suspending a $6 \mathrm{~mm}$. diameter eircular halfwave plate horizontally from a quartz fibre with a torsional constant of $2 \times 10^{-8}$ dyne $\mathrm{em}$. per radian, and allowing circularly polarised light to traverse it, thus reversing the direction of rotation of the electromagnetic light-vectors, we were able to observe torques of amounts plus and minus $2 \times 10^{-11}$ dyne cm., according to the direction of rotation of the incident light. The circular polarisation of the incident light was produced by passing plane polarised light through a quarter-wave plate. By rotating this quarter-wave plate through $360^{\circ}$, the light could be polarised twice in a clockwise direction and twice anti-clockwise.

Different observations of the torque were consistent to 3 per cent. If the angular momentum of elliptically polarised light is $k \frac{E}{2 \pi \nu} \sin \gamma$, where $E$ is its energy, $v$ its frequency and $\gamma$ the phase difference of the equal and perpendicular vibrations composing it, then from our experiments $k=1.05 \pm 0.15$, or the angular momentum of one quantum of circularly polarised light is $h / 2 \pi$ within the experimental error. The chief experimental uncertainty at present is in the determination of $E$.

This confirms the qualitative results obtained by R. A. Beth ${ }^{1}$ in September.

A. H. S. Holbourn.

Clarendon Laboratory, Oxford.

Dec. 13.

Phys. Rex., 48, No. 5, 471 (1935).

\section{Low-Temperature Masking of Tobacco Mosaic Symptoms}

Severar plants, when infected with an appropriate virus, fail to show symptoms upon new growth made during a period of rolatively high temperature. Thus leaves of tobacco infected with mosaic (tobacco virus 1 of Johnson), when grown above $98^{\circ} \mathrm{F}$., show no appearance of the disease. This is known as 'masking', and was first described by Prof. James Johnson ${ }^{1}$ in 1921. Most workers on plant viruses realise that the appearance of symptoms is delayed at cool temperatures; but an actual masking below a temperature of about $51^{\circ} \mathrm{F}$. can be demonstrated.

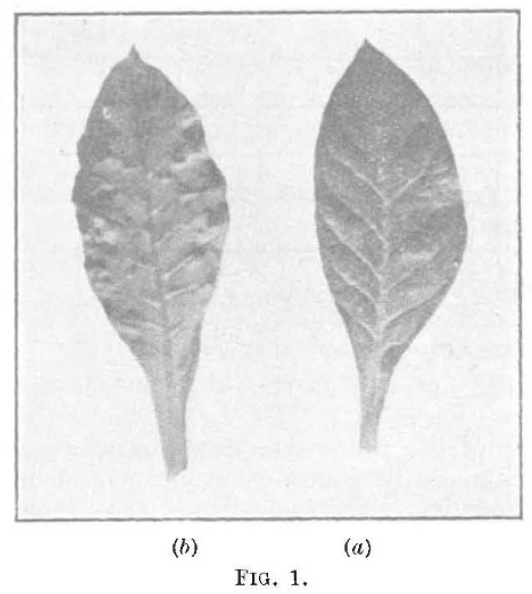

Six young tobacco plants showing mosaic symptoms were placed in a cool greenhouse on October 5, 1934. Growth was very slow, but six weeks later, on November 16, each had formed about three newly. expanded leaves, which wero all unmottled, and to all appearances healthy (Fig. $1 a$ ). These plants were then placed in a glass chamber maintained at a temperature of $75^{\circ} \mathrm{F}$., with full light. Within seven days, symptoms appeared on all the young leaves (Fig. 1b) made above the masked leaves. The latter remained seemingly healthy. All the plants were afterwards removed to the cool house, and by Christmas 1934 had made further symptom-free growth. The cool greenhouse maintained a temperature of $51^{\circ} \mathrm{F} . \pm 2^{\circ}$, except during bright sunlight. This was of short duration in Huddersfield, during the period of the experiment, and the maximum temperature was $57^{\circ} \mathrm{F}$. Masking could not be due to the effects of low light intensity, since ten diseased tobacco plants in a neighbouring house at $65^{\circ} \mathrm{F}$., and with similar light conditions, maintained their symptoms on all leaves throughout the time of the experiment.

The trial has been repeated this year with ten diseased plants, five at an average temperature of $55^{\circ} \mathrm{F}$. and five at $45^{\circ} \mathrm{F}$. The higher temperature did not completely mask the appearance of symptoms, for one or two slightly-mottled areas often appeared on a leaf. Plants grown at the temperature of $45^{\circ} \mathrm{F} . \pm 3^{\circ}$ showed completely masked symptoms.

It appears, therefore, that the symptoms of tobacco mosaic are masked when the host plant is grown at a temperature below about $5 \mathrm{I}^{\circ} \mathrm{F}$.

A preliminary attempt to compare the optimum temperature of growth of the plant with that for the greatest activity of the virus seems to indicate that the plant grows quickest at $75^{\circ} \mathrm{F}$. under Huddersfield 